\title{
LINC00294 induced by GRP78 promotes cervical cancer development by promoting cell cycle transition
}

\author{
JIANGNAN QIU*, SHULIN ZHOU*, WENJUN CHENG and CHENGYAN LUO \\ Department of Gynecology, The First Affiliated Hospital of Nanjing Medical University, Nanjing, Jiangsu 210000, P.R. China
}

Received May 24, 2018; Accepted May 27, 2020

DOI: $10.3892 / \mathrm{ol} .2020 .12125$

\begin{abstract}
Cervical cancer is one of the most common gynecological malignancies, and it has become a crucial public health problem. In the present study, the expression profiles of cervical cancer and normal cervical tissues were downloaded from the Gene Expression Omnibus and The Cancer Genome Atlas databases. Subsequently, the dysregulated long non-coding RNAs (IncRNAs) in cervical cancer were identified using $\mathrm{R}$ software Differentially expressed lncRNAs in cervical cancer that were associated with glucose-regulated protein 78 (GRP78) were screened out and the results demonstrated that eight lncRNAs were strongly positively correlated with GRP78. In order to confirm the relationship between GRP78 and candidate 1ncRNAs, GRP78 small interfering RNA (siRNA) was transfected into HeLa cells. The target IncRNAs that were regulated by GRP78 were then identified by reverse transcription-quantitative PCR and it was revealed that LINC00294 was significantly downregulated following GRP78-knockdown. Subsequently, Gene Set Enrichment Analysis demonstrated that LINC00294 was mainly enriched in regulating the cell cycle and the Hedgehog pathway. Following transfection of HeLa and SiHa cells with LINC00294 siRNA, the cell cycle was arrested at the G0/G1 phase. Western blotting suggested that LINC00294-knockdown downregulated the expression of cell cycle-associated factors (cyclin D, cyclin E and cyclin Dependent kinase 4) and upregulated cell cycle inhibitory factors (p16 and p21). The Hedgehog pathway was inhibited following knockdown of LINC00294 in HeLa and SiHa cells. In summary, LINC00294 induced by GRP78 promoted the progression of cervical cancer by regulating the cell cycle via Hedgehog pathway.
\end{abstract}

Correspondence to: Dr Wenjun Cheng or Dr Chengyan Luo, Department of Gynecology, The First Affiliated Hospital of Nanjing Medical University, 300 Guangzhoulu, Nanjing, Jiangsu 210000, P.R. China

E-mail: wenjunchengdoc@163.com

E-mail: lcywei0209@163.com

${ }^{*}$ Contributed equally

Key words: cervical cancer, glucose-regulated protein 78, LINC00294, cell cycle, Hedgehog pathway

\section{Introduction}

Cervical cancer is one of the most common gynecological malignancies, and it has become a crucial public health problem (1). Globally, the incidence of cervical cancer ranks second among all female malignant tumors (2). At present, surgery, chemotherapy and radiotherapy are the preferred treatments for cervical cancer. However, chemotherapy drugs cannot achieve a good therapeutic effect in cervical cancer due to drug resistance. In addition, effective treatments for advanced and recurrent cervical cancer are lacking (3). However, targeted therapy for cervical cancer has been well recognized. Specific molecular targeted drugs for tumors have gradually been identified, such as drugs that target signal transduction, cell receptors and angiogenesis $(4,5)$. To the best of our knowledge, the exact mechanism of cervical cancer development is rarely studied, which greatly limits the investigation of molecular targeted drug therapy. Therefore, it is necessary to further study the molecular mechanism of cervical cancer development.

With the progress of human genome sequencing technology, $>98 \%$ of sequences in the human genome are found to be non-coding RNAs (6) Non-coding RNAs are classified into short and long non-coding RNAs (lncRNAs) based on their sequence lengths (7). IncRNAs are $>200$ nucleotides in length and their structure is similar to mRNAs $(8,9)$. IncRNA is ubiquitous in eukaryotes, however its sequence is poorly conserved among different species (10). IncRNAs can form complex secondary structures that provide space for binding to multiple nucleic acids or proteins (11). Functionally, lncRNA regulates gene expression at the transcriptional and post-transcriptional level (12). IncRNAs are also involved in a variety of cellular activities, such as signaling pathway regulation, gene expression regulation, protein folding and cell activity (13). Studies have also reported that IncRNAs are closely associated various tumors, such as colorectal cancer and breast cancer (14-16).

Glucose-regulated protein 78 (GRP78) is a multifunctional protein that is mainly distributed in the endoplasmic reticulum (17). GRP78 acts as a molecular chaperone that controls protein folding and assembly, prevents protein aggregation and regulates the endoplasmic reticulum unfolded protein response pathway (18). As an essential stress sensor, GRP78 expression is altered by various factors in the tumor microenvironment, such as hypoxia, glucose and nutrient deficiency, acidosis, and inflammatory responses (19). A number 
of studies have shown that GRP78 overexpression promotes proliferation, migration, invasion and anti-apoptosis of cancer cells $(20,21)$. GRP78-specific antibody is capable of inhibiting tumor growth and metastasis by neutralizing GRP78 protein level (22). Our previous studies demonstrated that GRP78 is upregulated in cervical cancer, and GRP78-knockdown could increase the sensitivity of chemotherapy drugs and improve the cisplatin-induced apoptosis of cervical cancer cells $(23,24)$.

In the present study, The Cancer Genome Atlas (TCGA) and Gene Expression Omnibus (GEO) databases were used to screen target lncRNAs that were associated with GRP78 in cervical cancer. Subsequent experiments were performed to investigate the underlying mechanism.

\section{Materials and methods}

Data collection. Two cervical cancer expression microarrays GSE26511 (https://www.ncbi.nlm.nih.gov/geo/query/acc. cgi?acc=gse26511) and GSE5787 (https://www.ncbi.nlm.nih. gov/geo/query/acc.cgi) were downloaded from the GEO database. The GSE26511 microarray contains 20 cases of cervical cancer without lymph node metastasis and 19 cases of cervical cancer with lymph node metastasis (25). GSE5787 contain 30 tumor samples from 11 patients with cervical cancer (26). TCGA http://cancergenome.nih.gov/) contained gene expression profile data of cervical cancer. All data from TCGA were downloaded in December 2017, and consisted of data for a total of 306 cervical cancer samples.

Data processing. Correlation analysis was performed between GRP78 and lncRNAs extracted from GSE5787, GSE26511 and TCGA with the Cor R package by R software. The top 200 lncRNAs with positive and negative correlation with GRP78 in GSE26511, GSE5787 and TCGA were extracted and analyzed in a Venn diagram (https://bioinfogp.cnb.csic. es/tools/venny/). Gene Set Enrichment Analysis (GSEA) was conducted using GSEA 2.2.1 software $(27,28)$. The downloaded expression profile, phenotype data and MsigDB microarray platform file were uploaded into the GSEA program. Enrichment analysis was performed according to the default weighted enrichment statistics method.

Cell culture and transfection. Human cervical cancer cell lines $\mathrm{HeLa}$ and SiHa were obtained from American Type Culture Collection. HeLa and SiHa cells were cultured in Dulbecco's modified Eagle's medium (Hyclone; Cytiva) containing 10\% fetal bovine serum (Hyclone; Cytiva), $100 \mathrm{U} / \mathrm{ml}$ penicillin and $100 \mu \mathrm{g} / \mathrm{ml}$ streptomycin (Hyclone; Cytiva). Cells were incubated in a $5 \% \mathrm{CO}_{2}$ incubator at $37^{\circ} \mathrm{C}$.

For transfection, HeLa were seeded in the 6-well plates, and when the confluence was $60-80 \%$, the cells were transfected with $50 \mathrm{nM}$ small interfering RNAs (siRNAs and si-NC used as control) using Lipofectamine 2000 (Invitrogen; Thermo Fisher Scientific, Inc.) according to the manufacturer's protocol. The culture medium was replaced $6 \mathrm{~h}$ later. After $24 \mathrm{~h}$, the transfected cells were harvested for subsequent experiments. The sequences of siRNA used were as follows: si-negative control (NC) sense, 5'-UUCUCCGAACGUGUCACGUTT-3' and antisense, 5'-ACG UGACACGUUCGGAGAATT-3'; si-GRP78 sense, 5'-GGA GCGCAUUGAUACUAGATT-3' and antisense, 5'-UCUAGU
AUCAAUGCGCUCCTT-3'; si-LINC00294-1 sense, 5'-CCA CAAAGUUAUCAGGAAATT-3' and antisense, 5'-UUUCCU GAUAACUUUGUGGTT-3'; si-LINC00294-2 sense, 5'-CCU GGAAUCUCAUAGGAUUTT-3' and antisense, 5'-AAUCCU AUGAGAUUCCAGGTT-3'; and si-LINC00294-3 sense, 5'-GCGAACAUGUAACCCUCUATT-3' and antisense, 5'-UAG AGGGUUACAUGUUCGCTT-3'.

$R N A$ extraction and reverse transcription-quantitative $P C R$ $(R T-q P C R)$. Total RNA was extracted from transfected cells using TRIzol (Invitrogen; Thermo Fisher Scientific, Inc.) and reversed transcribed using PrimeScript RT Master mix (Takara Bio, Inc.) according to the manufacturer's protocol. RNA concentration was detected using a spectrometer and samples with an A260/A280 ratio of 1.8-2.0 were selected for the following qPCR reaction. qPCR was then performed using $\mathrm{SYBR}^{\circledR}$ Green Master mix (Takara Bio, Inc.), according to the manufacturer's protocol. RT-qPCR was carried out at $94^{\circ} \mathrm{C}$ for $5 \mathrm{~min}$ and 40 cycles at $94^{\circ} \mathrm{C}$ for $30 \mathrm{sec}, 55^{\circ} \mathrm{C}$ for $30 \mathrm{sec}$ and $72^{\circ} \mathrm{C}$ for $90 \mathrm{sec}$ following the protocol of SYBR Premix Ex $\mathrm{Taq}^{\mathrm{TM}}$ (Takara). The primers used for $\mathrm{qPCR}$ were as follows: GAPDH forward, 5'-CACCCACTCCTCCACCTTTG-3' and reverse, 5'-CCACCACCCTGTTGCTGTAG-3'; HLA complex 5 forward, 5'-CTGTGGATGACATGGCCTTA-3' and reverse, 5'-GATGCCAGCTTTGAGTGGA-3'; HOXA transcript antisense RNA (HOTAIR), myeloid specific 1 forward, 5'-AGTGCTGGAGCGAAGAAGAG-3' and reverse, 5'-CCTCTCGCCAGTTCATCTTT-3'; HOXA11 antisense RNA forward, 5'-CGGCTAACAAGGAGATTTGG-3' and reverse, 5'-GCAAAGGCTGTGGAAAGAAG-3'; LINC00162 forward, 5'-CTCAGAAACACCCACCATGC-3' and reverse, 5'-GAAAGTCCAGGCAGTTCAGC-3'; LINC00294 forward, 5'-TGTGTTGTCCTCCAGAATCG-3' and reverse, 5'-CCA ACCAAGAGCCAACAAAG-3'; LINC00888 forward, 5'-TTG GCCCTTGAAAGAATCAG-3' and reverse, 5'-ACTGGCATT TCCTCCACTGT-3'; ST3GAL4 antisense RNA 1 forward, 5'-ACCTTCCTGCTGTCCTTCCT-3' and reverse, 5'-CCC TCAGAGCCTTGATGTCT-3'; TOPORS antisense RNA 1 forward, 5'-CTCTCACCTCCTGTTGTACGC-3' and reverse, 5'-CGTGGTCTGTTTAGGGAGGA-3'; P16 forward, 5'-TTA TTTGAGCTTTGGTTCTG-3' and reverse, 5'-CCGGCTTTC GTAGTTTTCAT-3'; P21 forward, 5'-GAGTCTCCAGGT CCACCTGG-3' and reverse, 5'-CGTGGTCTGTTTAGG GAGGA-3'; cyclin D forward, 5'-ATGTTCGTGGCCTCT AAGATGA-3' and reverse, 5'-CAGGTTCCACTTGAGCTT GTTC-3'; cyclin E forward, 5'-GTTATAAGGGAGACGGGG AG-3', and reverse, 5'-TGCTCTGCTTCTTACCGCTC-3'; and cyclin-dependent kinase 4 (CDK4) forward, 5'-CATGTA GACCAGGACCTAAGG-3', and reverse, 5'-AACTGGCGC ATCAGATCCTAG-3'. Relative gene expression was analyzed using $2^{-\Delta \Delta C t}$ method (29).

Cell cycle detection. HeLa and SiHa cells were digested with trypsin (Gibco; Thermo Fisher Scientific, Inc.) and prepared into a cell suspension. After cells were washed with Hank's buffer (Gibco; Thermo Fisher Scientific, Inc.), cells were centrifuged at $1,000 \mathrm{x}$ g for $5 \mathrm{~min}$ at room temperature. Cells were then resuspended and incubated with pre-cooled $70 \%$ ethanol overnight. Finally, cells were stained with propidium iodide $(30 \mu \mathrm{g} / \mathrm{ml}$ ) for $30 \mathrm{~min}$ at room temperature (Beyotime Institute 

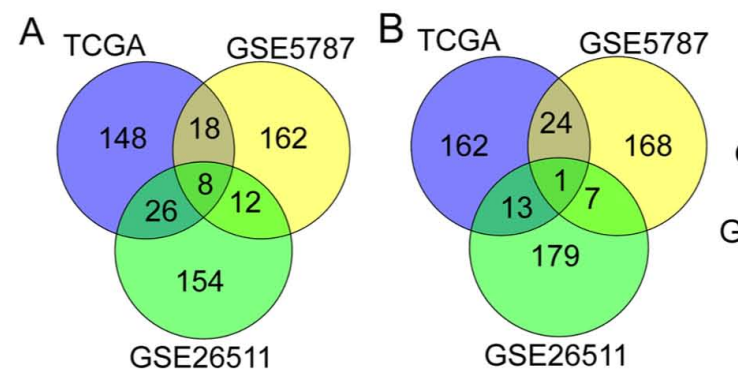

C $\mathrm{D}$
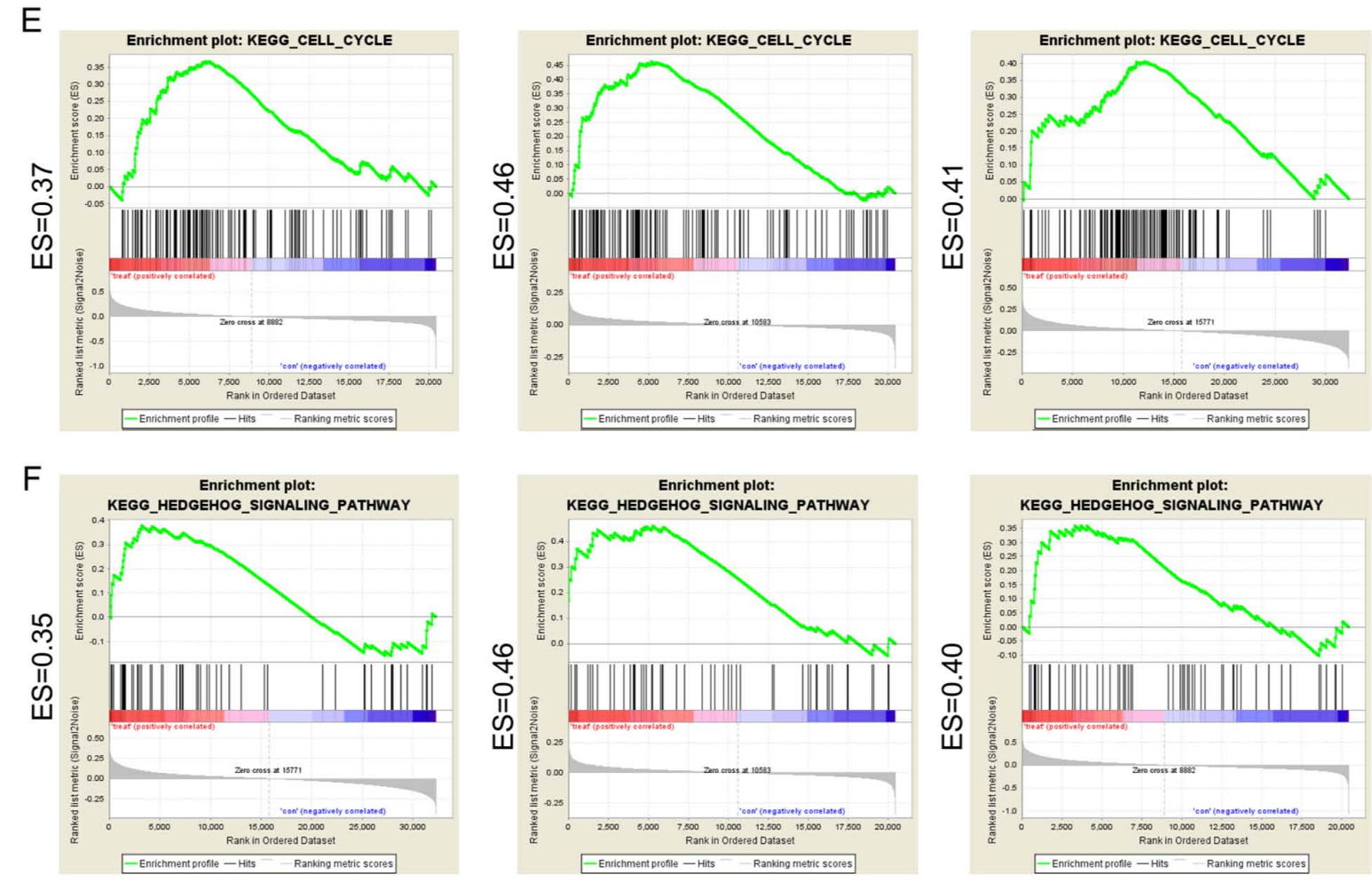

GSE5787 $(n=30)$

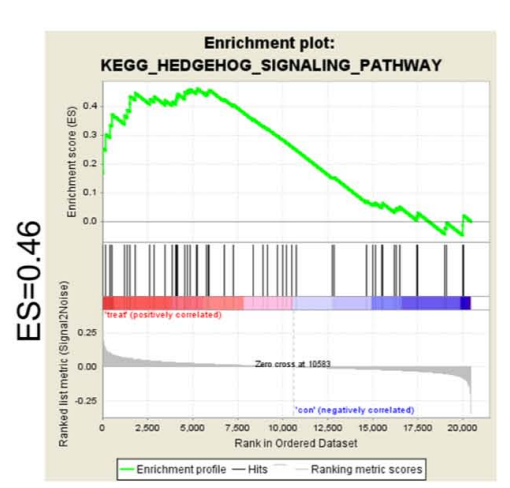

GSE26511 $(n=39)$

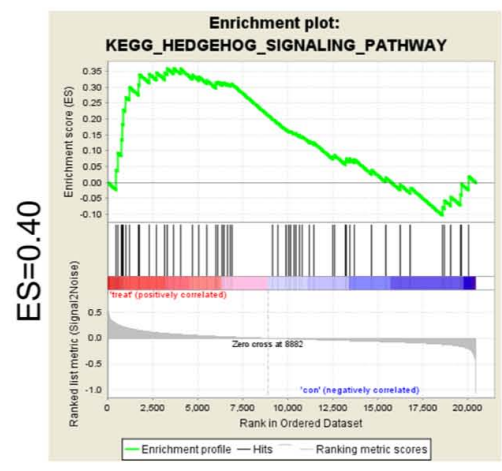

$\operatorname{TCGA}(n=306)$

Figure 1. GRP78 regulates LINC00294 expression. (A) The top 200 lncRNAs that were positively correlated with GRP78 in the GSE26511, GSE5787 and TCGA datasets. (B) The top 200 lncRNAs that were negatively correlated with GRP78 in the GSE26511, GSE5787 and TCGA datasets. (C) The GRP78 protein expression level was decreased by transfection with si-GRP78. (D) LINC00294 expression was decreased following GRP78-knockdown. (E) LINC00294 is associated with the cell cycle according to GSEA. (F) GSEA demonstrated that LINC00294 is enriched in the Hedgehog pathway. ${ }^{* * *} \mathrm{P}<0.001$. GRP78, glucose-regulated protein 78; lncRNA, long non-coding RNA; TCGA, The Cancer Genome Atlas; si, small interfering RNA; NC, negative control; HCP5, HLA complex 5; HOTAIRM1, HOXA transcript antisense RNA, myeloid specific 1; HOXA11-AS, HOXA11 antisense; ST3GAL4-AS1, ST3GAL4 antisense RNA 1; TOPORs-AS1, TOPORS antisense RNA 1; ES, enrichment score; KEGG, Kyoto Encyclopedia of Genes and Genomes; GSEA, Gene Set Enrichment Analysis.

of Biotechnology), followed by cell cycle detection using a flow cytometer (BD LSRFFortessa; BD Biosciences). The results were analyzed using FlowJo software (version 10.6.2; BD Biosciences).

Western blotting. Total protein was extracted from treated cells using RIPA solution at $4^{\circ} \mathrm{C}$ (Beyotime Institute of Biotechnology) and the protein concentration was quantified using BCA kit (Beyotime Institute of Biotechnology). Protein samples $(60 \mu \mathrm{g})$ were separated by $10 \%$ SDS gel with electrophoresis and transferred to a PVDF membrane. Membranes were blocked with 5\% skimmed milk for $1 \mathrm{~h}$ at room temperature, followed by incubation with the primary antibodies against GRP78 (1:1,000; cat. no. 3177T), p16 (1:1,000; cat. no. 80772), p21 (1:1,000; cat. no. 2947), Cyclin D (1:1,000; cat. no. 55506), Cyclin E (1:1,000; cat. no. 4129), CDK4 (1:1,000; cat. no. 12790), Gli1 (1:1,000; cat. no. 3538T),
Sonic (1:1,000; cat. no. 2207T) and GAPDH (1:1,000; cat. no. 5174T; Cell Signaling Technology, Inc.) overnight at $4^{\circ} \mathrm{C}$. Subsequently, the membranes were incubated with anti-rabbit IgG HRP-linked antibody (1:1,000; cat. no. 7074; Cell Signaling Technology, Inc.) at room temperature for $1 \mathrm{~h}$. The protein blots on the membrane were exposed by chemiluminescence reagent (Thermo Fisher Scientific, Inc.). Relative expression levels were normalized to endogenous control GAPDH using Image J software (1.52a; National Institutes of Health).

Statistical analysis. All experiments were performed in triplicate. SPSS 20.0 statistical software (IBM Corp.) was used for statistical analysis, and GraphPad 5.0 (GraphPad Software, Inc.) was used to generate figures. Quantitative data are expressed as mean \pm standard deviation. Comparisons among multiple groups were performed by one-way analysis of 

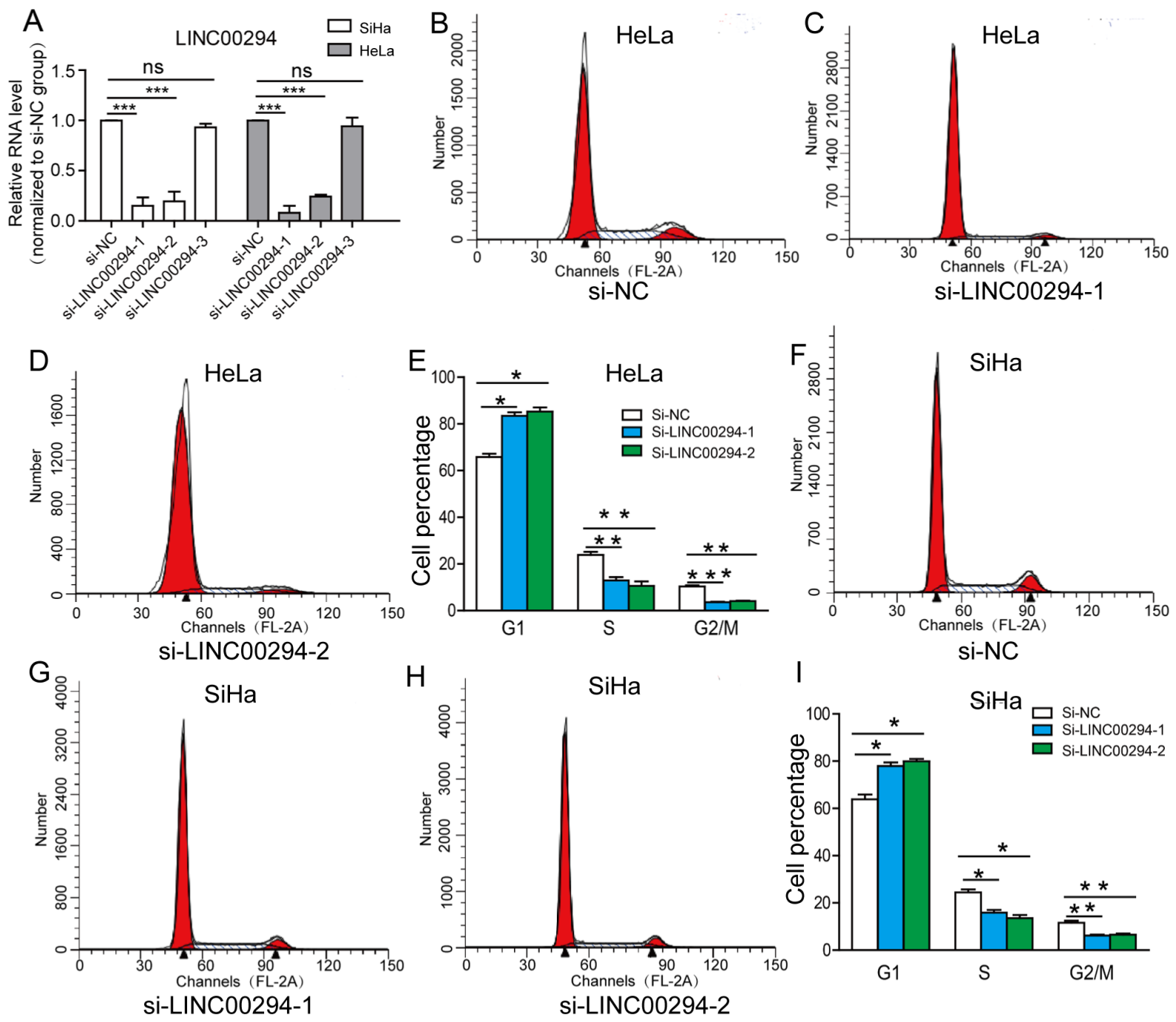

Figure 2. LINC00294-knockdown arrests the cell cycle at the G0/G1 phase. (A) Transfection of HeLa and SiHa cell with si-LINC00294. (B-E) The cell cycle of HeLa cells was arrested at the G0/G1 phase following LINC00294-knockdown. (F-I) Cell cycle of SiHa cells was arrested at the G0/G1 phase following LINC00294 knockdown. ${ }^{*} \mathrm{P}<0.05,{ }^{* *} \mathrm{P}<0.01,{ }^{* * *} \mathrm{P}<0.001$. si, small interfering RNA; NC, negative control; ns, not significant.

variance. Comparisons among four groups were analyzed by one-way analysis of variance followed by Tukey's post hoc test. $\mathrm{P}<0.05$ was considered to indicate a statistically significant difference.

\section{Results}

GRP78 regulates LINC00294 expression. Cervical cancer gene expression profile data were downloaded from GEO and TCGA databases. Specifically, GSE26511 contained 39 cervical cancer samples, GSE5787 contained 30 cervical cancer samples and TCGA contained 306 cervical cancer samples. Subsequently, correlation analysis between the identified lncRNAs and GRP78 was performed, the top 200 lncRNAs that were positively correlated with GRP78 and the top 200 lncRNAs that were negatively correlated with GRP78 were selected. The overlapping lncRNAs that were positively correlated with GRP78 in the GSE26511, GSE5787 and TCGA datasets were obtained from the Venn diagram. A total of eight overlapping lncRNAs were finally screened out to be positively correlated with GRP78 (Fig. 1A). Whereas, only one lncRNA was negatively correlated with GRP78 in all datasets (Fig. 1B). These eight positively corrected lncRNAs (LINC00294, HCP5, HOTAIRM1, HOXA11-AS, LINC00162, LINC00888) were selected for the following experiments.

For the following experiments, si-GRP78 was transfected into HeLa cells and the expression levels of the eight positively correlated lncRNAs were detected. Western blotting was used to confirm that GRP78 was successfully reduced in HeLa cells transfected with si-GRP78 compared with cells transfected with the si-NC (Fig. 1C). Subsequently, the eight positively corrected IncRNAs were confirmed by RT-qPCR following GRP78 knockdown. The results demonstrated that only LINC00294 was downregulated following GRP78-knockdown in HeLa cells (Fig. 1D). Subsequently, GSEA was performed to predict the biological processes that LINC00294 is associated with. The results demonstrated that the LINC00294 is associated with the cell cycle (Fig. 1E) and Hedgehog signaling pathway (Fig. 1F). These results indicated that LINC00294 may participate in cervical cancer development via GRP78. 
A

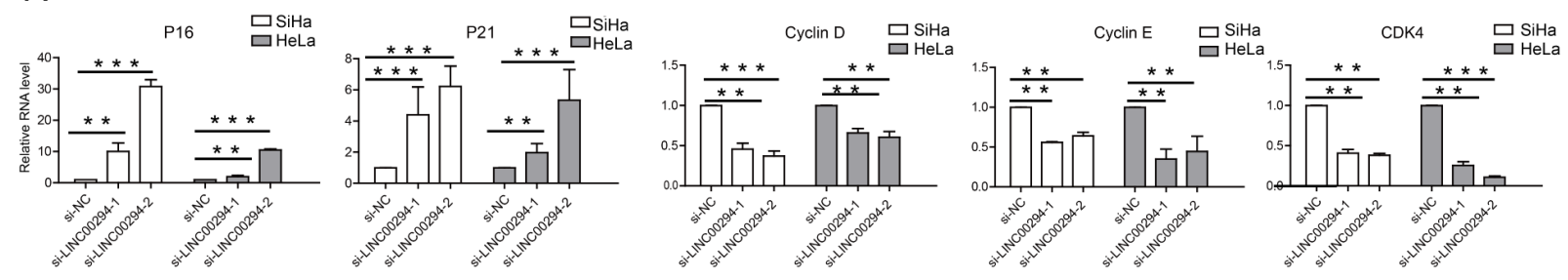

B

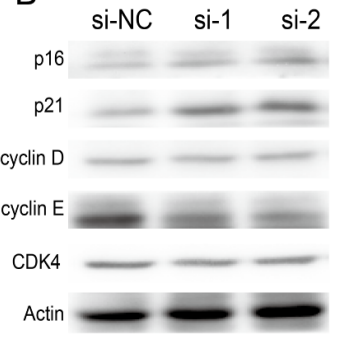

$\mathrm{SiHa}$

D

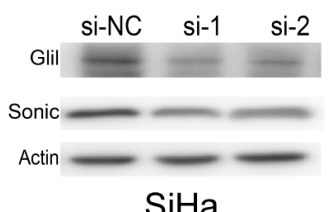

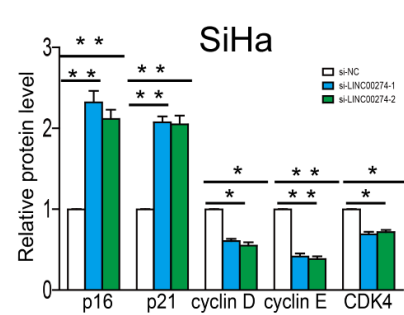

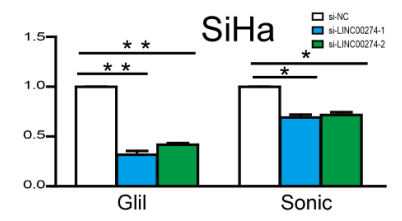

C

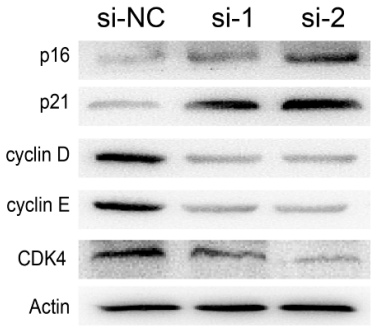

HeLa

E

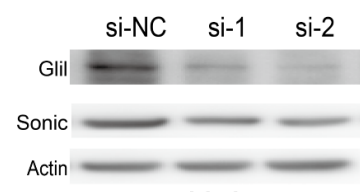

HeLa
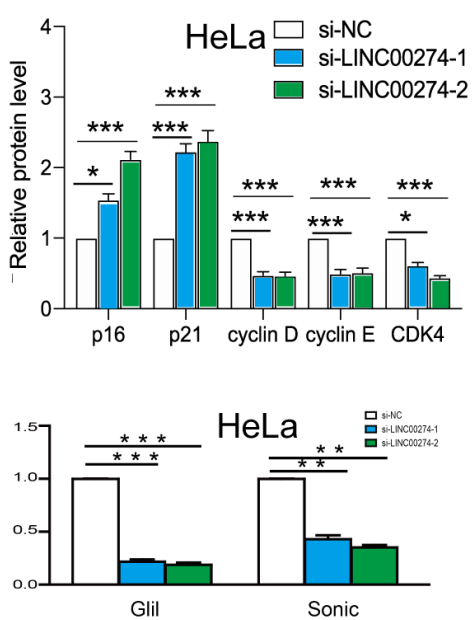

Figure 3. LINC00294 regulates the cell cycle via the Hedgehog pathway. (A) The mRNA levels of p16, p21, cyclin D, cyclin E and CDK in HeLa and SiHa cells that were transfected with si-LINC00294. The protein levels of p16, p21, cyclin D, cyclin E and CDK in (B) SiHa and (C) HeLa cells following LINC00294-knockdown. The protein levels of Sonic and Gli1 in (D) SiHa and (E) HeLa cells following LINC00294-knockdown. ${ }^{*} \mathrm{P}<0.05,{ }^{* * *} \mathrm{P}<0.01,{ }^{* * *} \mathrm{P}<0.001$. si, small interfering RNA; NC, negative control; CDK4, cyclin-dependent kinase 4; Gli1, GLI family zinc finger 1.

LINC00294-knockdown arrests the cell cycle of cervical cancer cells. To investigate whether LINC00294 can regulate the cell cycle of HeLa and SiHa cells, three siRNA sequences targeting LINC00294 were transfected into the cells. It was identified that si-LINC00294-1 and si-LINC00294-2 significantly reduced the mRNA level of LINC00294, whereas the efficacy of si-LINC00294-3 was insignificant (Fig. 2A). Following transfection of HeLa and $\mathrm{SiHa}$ cells with si-LINC00294-1 and si-LINC00294-2, the percentage of cells in the G0/G1 phase was significantly increased, whereas the percentages of cells in the $\mathrm{S}$ and $\mathrm{G} 2 / \mathrm{M}$ phases were significantly decreased (Fig. 2B-D). These results demonstrated that LINC00294-knockdown arrests the cell cycle at the G0/G1 phase, thereby inhibiting the proliferative ability of cervical cancer cells.

LINC00294 regulates the cell cycle of cervical cancer cells via the Hedgehog pathway. Expression levels of cell cycle-related genes were detected by RT-qPCR and western blotting. The results indicated that following LINC00294-knockdown in $\mathrm{HeLa}$ and SiHa cells the mRNA levels of p16 and p21 were significantly increased compared with the control cells, whereas the mRNA levels of cyclin D, cyclin E and CDK4 were significantly decreased (Fig. 3A). Similar results were obtained by western blotting (Fig. 3B and C). Based on GSEA data, the Hedgehog pathway was predicted to be involved in cervical cancer development. Therefore, key genes in Hedgehog pathway were detected by western blotting. Protein expression levels of Sonic and Gli1 were significantly decreased following transfection with si-LINC00294 (Fig. 3D and E), indicating a role of the Hedgehog pathway in regulating the cell cycle of cervical cancer cells.

\section{Discussion}

Cervical cancer is one of the most common types of malignant tumor in women, and it is a continuous process from a benign lesion, cervical intraepithelial neoplasia, carcinoma in situ and invasive carcinoma (30). Therefore, early detection and treatment are essential to reduce the morbidity and mortality of cervical cancer (31). Whole genome sequencing results have demonstrated that the majority of the stably transcribed RNAs are non-coding RNAs (32). Among them, lncRNAs possess functions in multiple cellular processes, including cell proliferation, differentiation and metabolism (33). IncRNAs are involved in various diseases, such as tumors, diabetes and immune diseases $(34,35)$. For example, overexpressed HOTAIR in cervical cancer is associated with lymph node metastasis, survival rate and postoperative recurrence (36). Maternally expressed 3 is downregulated in cervical cancer tissues, which promotes proliferation of cervical cancer (37). In addition, decreased expression of IncRNA growth arrest specific 5 can serve as an unfavorable prognostic factor for cervical cancer (38). Our previous study demonstrated that 
GRP78 is upregulated in cervical cancer tissues compared with paracancerous tissues. In the present study, LINC00294 was identified to be positively associated with GRP78 in. In vitro experiments demonstrated that LINC00294 may regulate the cell cycle of cervical cancer cells via the Hedgehog pathway.

Cell cycle disorder is one of the main causes of tumorigenesis (39). Under normal circumstances, the cell cycle transitions between the G1, S, G2 and M phases via precise regulation by the cell cycle molecular network system of cyclins, CDKs and cyclin-depending kinase inhibitors (CKIs) (40). Specifically, cyclin D1, cyclin E, CDK4, p16 and p21 are key factors that regulate the cell cycle $(39,40)$. At different phases of the cell cycle, cyclins bind to the corresponding CDKs to form cyclin/CDK complexes, which activate CDKs and promote cell cycle transformation. However, CDKs or cyclin/CDK complexes also inhibit CDK activities via binding to the corresponding CKIs, thereby inhibiting the transition of the cell cycle $(39,40)$. The present study demonstrated that LINC00294-knockdown in cervical cancer significantly arrests cell cycle in G0/G1 phase, thereby inhibiting cell cycle progression and cell proliferation. Western blotting demonstrated that LINC00294-knockdown downregulated cyclin D, cyclin E and CDK4, whereas it upregulated p16 and p21 in HeLa and SiHa cells.

The Hedgehog gene was first discovered in drosophila in 1980 (41). Previous studies have reported that the Hedgehog pathway plays a key role in animal embryonic development, including in the formation of lungs, skin, bones, limbs, neural tube and the gastrointestinal system (42-44). In addition, the Hedgehog pathway is involved in the regulation of cell growth, proliferation, migration and differentiation $(45,46)$. Hedgehog is closely associated with the occurrence and progression of malignant tumors, such as basal cell carcinoma, breast cancer, prostate cancer and multiple digestive system cancers $(47,48)$. Abnormal activation of the Hedgehog pathway results in invasion and metastasis of tumor cells (49-51). In the present study, the Hedgehog pathway was found to be involved in the occurrence of cervical cancer. Preliminary mechanism studies demonstrated that LINC00294-knockdown inhibited the expression levels of Gli1 and Sonic, which are key genes in the Hedgehog pathway.

In summary, the present study first identified LINC00294 to be strongly correlated with GRP78. Subsequently, RT-qPCR demonstrated that LINC00294 was regulated by GRP78. In addition, GSEA revealed that LINC00294 was mainly enriched in mediating the cell cycle and the Hedgehog pathway. An in vitro assay confirmed that LINC00294 could regulate the cell cycle. Furthermore, western blotting demonstrated that following knockdown of LINC00294, the CKD family was significantly downregulated and CDK were inhibitors upregulated. Additionally, Hedgehog pathway-associated proteins were also expressed at lower levels following knockdown of LINC00294. The key findings of the present study were that LINC00294 was regulated by GRP78 and knockdown of LINC00294 could arrest the cell cycle at the G0/G1 phrase. However, there were some limitations of the study. In order to further confirm the regulatory relationship between GRP78 and LINC00294, whether overexpression of GRP78 can increase the expression of LINC00294 should be investigated, as well as the effects of LINC00294 on cell proliferation, invasion and migration using in vivo experiments, which will be performed in future studies. In conclusion, LINC00294 is positively correlated with and regulated by GRP78, which promotes the progression of cervical cancer through arresting the cell cycle at the G0/G1 phase via the Hedgehog pathway.

\section{Acknowledgements}

Not applicable.

\section{Funding}

This study was supported by the Jiangsu Maternal and Child Health Key Program (grant no. F2012110).

\section{Availability of data and materials}

The datasets used and/or analyzed during the current study are available from the corresponding author on reasonable request.

\section{Authors' contributions}

JQ and SZ performed the experiments and collected the data. WC analyzed the data. CL conceived and designed the experiments, and wrote the manuscript. All authors read and approved the final manuscript.

\section{Ethics approval and consent to participate}

Not applicable.

\section{Patient consent for publication}

Not applicable.

\section{Competing interests}

The authors declare that they have no competing interests.

\section{References}

1. Siegel RL, Miller KD and Jemal A: Cancer statistics, 2017. CA Cancer J Clin 67: 7-30, 2017.

2. Ferlay J, Shin HR, Bray F, Forman D, Mathers C and Parkin DM: Estimates of worldwide burden of cancer in 2008: GLOBOCAN 2008. Int J Cancer 127: 2893-2917, 2010.

3. Nunes de Arruda F, da Costa S, Bonadio R, Dornellas A, Pereira D, de Bock GH and Del Pilar Estevez Diz M: Quality of life of locally advanced cervical cancer patients after neoadjuvant chemotherapy followed by chemoradiation versus chemoradiation alone (CIRCE trial): A randomized phase II trial. Int J Gynecol Cancer 30: 749-756, 2020.

4. Diaz-Padilla I, Monk BJ, Mackay HJ and Oaknin A: Treatment of metastatic cervical cancer: Future directions involving targeted agents. Crit Rev Oncol Hematol 85: 303-314, 2013.

5. Zagouri F, Sergentanis TN, Chrysikos D, Filipits M and Bartsch R: Molecularly targeted therapies in cervical cancer. A systematic review. Gynecol Oncol 126: 291-303, 2012.

6. Djebali S, Davis CA, Merkel A, Dobin A,Lassmann T, Mortazavi A, Tanzer A, Lagarde J, Lin W, Schlesinger F, et al: Landscape of transcription in human cells. Nature 489: 101-108, 2012.

7. Esteller M: Non-coding RNAs in human disease. Nat Rev Genet 12: 861-874, 2011.

8. Marques AC and Ponting CP: Catalogues of mammalian long noncoding RNAs: Modest conservation and incompleteness. Genome Biol 10: R124, 2009. 
9. Ørom UA, Derrien T, Beringer M, Gumireddy K, Gardini A Bussotti G, Lai F, Zytnicki M, Notredame C, Huang Q, et al: Long noncoding RNAs with enhancer-like function in human cells. Cell 143: 46-58, 2010

10. Kutter C, Watt S, Stefflova K, Wilson MD, Goncalves A, Ponting CP, Odom DT and Marques AC: Rapid turnover of long noncoding RNAs and the evolution of gene expression. PLoS Genet 8: e1002841, 2012 .

11. Perez DS, Hoage TR, Pritchett JR, Ducharme-Smith AL, Halling ML, Ganapathiraju SC, Streng PS and Smith DI: Long, abundantly expressed non-coding transcripts are altered in cancer. Hum Mol Genet 17: 642-655, 2008.

12. Hung T and Chang HY: Long noncoding RNA in genome regulation: Prospects and mechanisms. RNA Biol 7: 582-585, 2010.

13. Yan Y, Zhang L, Jiang Y, Xu T, Mei Q, Wang H, Qin R, Zou Y, $\mathrm{Hu} \mathrm{G}$, Chen J and Lu Y: LncRNA and mRNA interaction study based on transcriptome profiles reveals potential core genes in the pathogenesis of human glioblastoma multiforme. J Cancer Res Clin Oncol 141: 827-838, 2015.

14. Liao Q, He W, Liu J, Cen Y, Luo L, Yu C, Li Y, Chen S and Duan S: Identification and functional annotation of 1 ncRNA genes with hypermethylation in colorectal cancer. Gene 572 259-265, 2015.

15. Richards EJ, Zhang G, Li ZP, Permuth-Wey J, Challa S, Li Y, Kong W, Dan S, Bui MM, Coppola D, et al: Long non-coding RNAs (LncRNA) regulated by transforming growth factor (TGF) $\beta$ : LncRNA-hit-mediated TGF $\beta$-induced epithelial to mesenchymal transition in mammary epithelia. J Biol Chem 290: 6857-6867, 2015

16. Wang R, Du L, Yang X, Jiang X, Duan W, Yan S, Xie Y, Zhu Y, Wang Q, Wang L, et al: Identification of long noncoding RNAs as potential novel diagnosis and prognosis biomarkers in colorectal cancer. J Cancer Res Clin Oncol 142: 2291-2301, 2016.

17. Ramirez MU, Hernandez SR, Soto-Pantoja DR and Cook KL: Endoplasmic reticulum stress pathway, the unfolded protein response, modulates immune function in the tumor microenvironment to impact tumor progression and therapeutic response. Int J Mol Sci 21: 169, 2019.

18. Bánhegyi G, Baumeister P, Benedetti A, Dong D, Fu Y, Lee AS Li J, Mao C, Margittai E, Ni M, et al: Endoplasmic reticulum stress. Ann N Y Acad Sci 1113: 58-71, 2007.

19. Li Z and Li Z: Glucose regulated protein 78: A critical link between tumor microenvironment and cancer hallmarks. Biochim Biophys Acta 1826: 13-22, 2012.

20. Misra UK, Payne S and Pizzo SV: Ligation of prostate cancer cell surface GRP78 activates a proproliferative and antiapoptotic feedback loop: A role for secreted prostate-specific antigen. J Biol Chem 286: 1248-1259, 2011.

21. Huang P, Guo Y, Zhao Z, Ning W, Wang H, Gu C, Zhang M, Qu Y, Zhang $\mathrm{H}$ and Song Y: UBE2T promotes glioblastoma invasion and migration via stabilizing GRP78 and regulating EMT. Aging (Albany NY) 12: 10275-10289, 2020.

22. Misra UK, Mowery Y, Kaczowka S and Pizzo SV: Ligation of cancer cell surface GRP78 with antibodies directed against its $\mathrm{COOH}$-terminal domain up-regulates $\mathrm{p} 53$ activity and promotes apoptosis. Mol Cancer Ther 8: 1350-1362, 2009.

23. Luo C, Fan W, Jiang Y, Zhou S and Cheng W: Glucose-related protein 78 expression and its effects on cisplatin-resistance in cervical cancer. Med Sci Monit 24: 2197-2209, 2018.

24. Luo C and Qiu J: miR-181a inhibits cervical cancer development via downregulating GRP78. Oncol Res 25: 1341-1348, 2017.

25. Noordhuis MG, Fehrmann RS, Wisman GB, Nijhuis ER van Zanden JJ, Moerland PD, van Themaat EVL, Volders HH, Kok M, ten Hoor KA, et al: Involvement of the TGF-beta and beta-catenin pathways in pelvic lymph node metastasis in early-stage cervical cancer. Clin Cancer Res 17: 1317-1330, 2011.

26. Bachtiary B, Boutros PC, Pintilie M, Shi W, Bastianutto C, Li JH, Schwock J, Zhang W, Penn LZ, Jurisica I, et al: Gene expression profiling in cervical cancer: An exploration of intratumor heterogeneity. Clin Cancer Res 12: 5632-5640, 2006

27. Mootha VK, Lindgren CM, Eriksson KF, Subramanian A Sihag S, Lehar J, Puigserver P, Carlsson E, Ridderstråle M, Laurila E, et al: PGC-1alpha-responsive genes involved in oxidative phosphorylation are coordinately downregulated in human diabetes. Nat Genet 34: 267-273, 2003.

28. Subramanian A, Tamayo P, Mootha VK, Mukherjee S, Ebert BL, Gillette MA, Paulovich A, Pomeroy SL, Golub TR, Lander ES and Mesirov JP: Gene set enrichment analysis: A knowledge-based approach for interpreting genome-wide expression profiles. Proc Natl Acad Sci USA 102: 15545-15550, 2005.
29. Livak KJ and Schmittgen TD: Analysis of relative gene expression data using real-time quantitative PCR and the 2(-Delta Delta C(T)) method. Methods 25: 402-440, 2001.

30. Polman NJ, Ebisch RMF, Heideman DAM, Melchers WJG, Bekkers RLM, Molijn AC, Meijer CJLM, Quint WGV, Snijders PJF, Massuger LFAG, et al: Performance of human papillomavirus testing on self-collected versus clinician-collected samples for the detection of cervical intraepithelial neoplasia of grade 2 or worse: A randomised, paired screen-positive, non-inferiority trial. Lancet Oncol 20: 229-238, 2019.

31. Halaska M, Robova H, Pluta M and Rob L: The role of trachelectomy in cervical cancer. Ecancermedicalscience 9: 506, 2015

32. Mercer TR, Dinger ME and Mattick JS: Long non-coding RNAs: Insights into functions. Nat Rev Genet 10: 155-159, 2009.

33. Yuan L, Xu ZY, Ruan SM, Mo S, Qin JJ and Cheng XD: Long non-coding RNAs towards precision medicine in gastric cancer: Early diagnosis, treatment, and drug resistance. Mol Cancer 19: 96, 2020.

34. Lee C and Kikyo N: Strategies to identify long noncoding RNAs involved in gene regulation. Cell Biosci 2: 37, 2012.

35. Novikova IV, Hennelly SP and Sanbonmatsu KY: Sizing up long non-coding RNAs: Do lncRNAs have secondary and tertiary structure? Bioarchitecture 2: 189-199, 2012.

36. Kim HJ, Lee DW, Yim GW, Nam EJ, Kim S, Kim SW and Kim YT: Long non-coding RNA HOTAIR is associated with human cervical cancer progression. Int J Oncol 46: 521-530, 2015.

37. Qin R, Chen Z, Ding Y, Hao J, Hu J and Guo F: Long non-coding RNA MEG3 inhibits the proliferation of cervical carcinoma cells through the induction of cell cycle arrest and apoptosis. Neoplasma 60: 486-492, 2013.

38. Cao S, Liu W, Li F, Zhao W and Qin C: Decreased expression of lncRNA GAS5 predicts a poor prognosis in cervical cancer. Int J Clin Exp Pathol 7: 6776-6783, 2014.

39. Murphy PJ and Campbell SS: Physiology of the circadian system in animals and humans. J Clin Neurophysiol 13: 2-16, 1996.

40. Lim S and Kaldis P: Cdks, cyclins and CKIs: Roles beyond cell cycle regulation. Development 140: 3079-3093, 2013.

41. Nusslein-Volhard C and Wieschaus E: Mutations affecting segment number and polarity in Drosophila. Nature 287: 795-801, 1980.

42. van Dop WA, Rosekrans SL, Uhmann A, Jaks V, Offerhaus GJ, van den Bergh WM, Kasper M, Heijmans J, Hardwick JC, Verspaget HW, et al: Hedgehog signalling stimulates precursor cell accumulation and impairs epithelial maturation in the murine oesophagus. Gut 62: 348-357, 2013

43. Zhang L, Yang Y, Liao Z, Liu Q, Lei X, Li M, Saijilafu, Zhang Z, Hong D, Zhu M, et al: Genetic and pharmacological activation of Hedgehog signaling inhibits osteoclastogenesis and attenuates titanium particle-induced osteolysis partly through suppressing the JNK/c-Fos-NFATc1 cascade. Theranostics 10: 6638-6660, 2020.

44. Sun Q, Zeng J, Liu Y, Chen J, Zeng QC, Chen YQ, Tu LL, Chen P, Yang F and Zhang M: microRNA-9 and -29a regulate the progression of diabetic peripheral neuropathy via ISL1-mediated sonic hedgehog signaling pathway. Aging (Albany NY) 12: 11446-11465, 2020.

45. BufalieriF,SeveriniLL,CaimanoM,InfantePand Di MarcotullioL: DUBs activating the hedgehog signaling pathway: A promising therapeutic target in cancer. Cancers (Basel) 12: 1518, 2020.

46. Lecca S, Namboodiri VMK, Restivo L, Gervasi N, Pillolla G, Stuber GD and Mameli M: Heterogeneous habenular neuronal ensembles during selection of defensive behaviors. Cell Rep 31: 107752, 2020.

47. Wu J, Zhu P, Lu T, Du Y, Wang Y, He L, Ye B, Liu B, Yang L, Wang J, et al: The long non-coding RNA LncHDAC2 drives the self-renewal of liver cancer stem cells via activation of Hedgehog signaling. J Hepatol 70: 918-929, 2019.

48. Hanna A and Shevde LA: Hedgehog signaling: Modulation of cancer properies and tumor mircroenvironment. Mol Cancer 15: 24, 2016.

49. Wang K, Pan L, Che X, Cui D and Li C: Sonic Hedgehog/GLI(1) signaling pathway inhibition restricts cell migration and invasion in human gliomas. Neurol Res 32: 975-980, 2010.

50. Onishi H, Kai M, Odate S, Iwasaki H, Morifuji Y, Ogino T, Morisaki T, Nakashima Y and Katano M: Hypoxia activates the hedgehog signaling pathway in a ligand-independent manner by upregulation of Smo transcription in pancreatic cancer. Cancer Sci 102: 1144-1150, 2011

51. Karhadkar SS, Bova GS, Abdallah N, Dhara S, Gardner D, Maitra A, Isaacs JT, Berman DM and Beachy PA: Hedgehog signalling in prostate regeneration, neoplasia and metastasis. Nature 431: 707-712, 2004.

This work is licensed under a Creative Commons Attribution-NonCommercial-NoDerivatives 4.0 International (CC BY-NC-ND 4.0) License. 